\begin{tabular}{|c|l|}
\hline Title & $\begin{array}{l}\text { Numerical analysis of spatial propagation of parametric fluorescence photon pairs using the tuning-curve filtering } \\
\text { method }\end{array}$ \\
\hline Author(s) & Fujiwara, Hideki; Kawabe, Yoshio; Takeuchi, Shigeki; Sasaki, Keiji \\
\hline Citation & $\begin{array}{l}\text { Physical Review. A, 75(2), 023802 } \\
\text { https://doi.org/_0.1103/PhysRevA.75.023802 }\end{array}$ \\
\hline Issue Date & 2007-02-01 \\
\hline Doc URL & http://hdl.handle.net/2115/33856 \\
\hline Rights & ○2007 The A merican Physical Society \\
\hline Type & article \\
\hline File Information & PhysRevA_75_023802.pdf \\
\hline
\end{tabular}

Instructions for use 


\title{
Numerical analysis of spatial propagation of parametric fluorescence photon pairs using the tuning-curve filtering method
}

\author{
Hideki Fujiwara, ${ }^{1,2, *}$ Yoshio Kawabe, ${ }^{1, \dagger}$ Shigeki Takeuchi, ${ }^{1,2, \$}$ and Keiji Sasaki ${ }^{1,2, \S}$ \\ ${ }^{1}$ Research Institute for Electronic Science, Hokkaido University, Sapporo 060-0812, Japan \\ ${ }^{2}$ CREST, Japan Science and Technology Agency (JST), Sapporo 060-0812, Japan
}

(Received 25 October 2006; published 1 February 2007)

\begin{abstract}
A method to quantitatively evaluate the propagation of parametric fluorescence is proposed. The probability distribution of photon pairs, including the influence of the optical setup in accordance with the wave vector and frequency, is numerically calculated. From this probability distribution, the coincidence counting probability can be simply evaluated. Comparing with experimental data of the iris size dependences of single and coincidence counting rates, the calculated results reproduce the experimental data well without the use of fitting parameters.
\end{abstract}

DOI: 10.1103/PhysRevA.75.023802

PACS number(s): 42.50.Dv

\section{INTRODUCTION}

Novel technologies using quantum-correlated photon pairs have been attracting attention recently [1-6]. For example, quantum lithography has the potential to achieve high spatial resolution beyond the Rayleigh diffraction limit [5]. In order to realize these technologies, a source that emits photon pairs with high yields and in well-defined modes is critical. For such photon-pair generation, the spontaneous parametric down-conversion (SPDC) process in a nonlinear crystal has been widely used [6-9]. However, in the experiments, it is necessary to efficiently collect the generated photon-pairs from the crystal to the targets (detectors or twophoton absorbers) passing through optical components such as filters, lenses, and apertures. For this purpose, the propagation of photon pairs through the optical system has been investigated [10-13]. Two approaches, geometrical and analytical, have been used primarily.

As an example of the geometrical approach, Pittman et al. performed a series of imaging experiments that demonstrated the two-photon geometric optics effect by taking advantage of momentum entanglement $[10,11]$. Imaging in coincidence counts of an aperture placed in one of the down-conversion beams was found to be the equivalent of classical geometric optics. The advantage of this method is that the propagation of photon pairs is intuitively comprehensible. However, similar to typical geometric optics, it is difficult to discuss the counting rates quantitatively.

As an example of the analytical approach, Joobeur et al. derived analytical expressions for the fourth-order spatiotemporal coherence function, which is proportional to the coincidence rate $[12,13]$. Using these expressions, they quantitatively discussed the coincidence rate of far-field downconverted light. However, such an analytical approach generally requires specific assumptions in order to solve the problem. Therefore if the method is applied for a different

\footnotetext{
*Email address: fuji@es.hokudai.ac.jp

†Email address: kawabe@es.hokudai.ac.jp

*Email address: takeuchi@es.hokudai.ac.jp

${ }^{\S}$ Email address: sasaki@es.hokudai.ac.jp
}

purpose, a major modification may be necessary. In addition, the analytical approach is not intuitively comprehensible.

In the present paper, we propose a different method to evaluate the propagation of photon pairs (single and coincidence counting rates) through optical components using numerical analysis. Boeuf et al. proposed a method of numerically determining the phase-matching configurations in both uniaxial and biaxial crystals [14]. They numerically calculated the dependence of the phase-matching function, which is proportional to the single counting rate, on the wavelength and output angles (tuning curve). Based on their research, we have developed a numerical method to quantitatively evaluate the single counting rate of photons passing through optical components, such as frequency filters and apertures. In the proposed method, the influence of each optical component is taken as a transmittance function in the frequency domain or in the wave-vector domain. Multiplying these transmittance functions ("filters") by the phase-matching function ("tuning curve") yields the detection probabilities.

Furthermore, we expand our method to evaluate the coincidence counting rate of the photon pairs through the optical components. We derive the conditional single counting probability considering the correlation in the phase-matching function. Then, we confirmed that the proposed method successfully reproduced the experimental data without any fitting parameters. With our method, the photon pairs through optical components can be analyzed visually and intuitively. Although our method is applicable to the type-I SPDC process [15], here we concentrate on the type-II SPDC process.

The paper is organized as follows. In Sec. II, we explain the concept of the tuning-curve filtering method. We describe the experimental setup for single and coincidence counting rates in Sec. III. In Sec. IV, experimental results are compared with the calculated results obtained using the proposed method, before conclusions in Sec. V.

\section{TUNING-CURVE FILTERING METHOD}

In this section, we describe the basic concept of our method and explain how the single and coincidence counting probabilities after the photons pass through the optical components can be evaluated. 


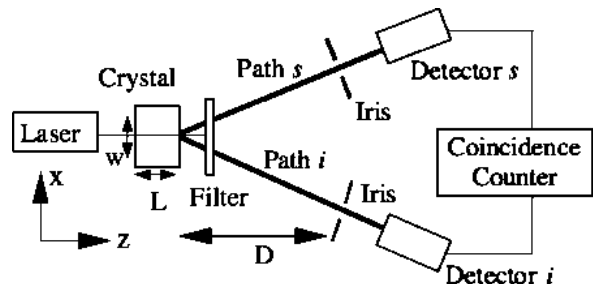

FIG. 1. Model of the optical system used for the calculations. A monochromatic excitation light with a Gaussian profile (width $w$ ) propagating along the $z$ axis is incident onto a beta-barium borate (BBO) crystal with a thickness of $L$. The crystal produces photon pairs (signal and idler photons) that satisfy the phase-matching condition of the type-II SPDC. The generated photons propagate through the optical components (interference filters and irises) set in each path (paths $s$ and $i$ ) at a distance of $D$ from the crystal. All photons passing through the optical components are detected by photodetectors.

\section{A. Tuning-curve filtering method}

Here, we concentrate on the type-II SPDC $[7,9,16]$. Figure 1 shows the model used in the following analysis. We ignore the walk-off effect in the crystal for the photon pairs. We define the $x-z$ plane to include the propagation axis of the excitation light and the optical axis of the crystal. The angle of the crystal axis with respect to the $z$ axis is denoted as $\theta_{p}$.

When multiphoton-pair emission can be ignored, the state $|\psi\rangle$ of the generated photon pairs from the crystal is given by $[13,17]$

$$
|\psi\rangle=|0\rangle+\int d \boldsymbol{k}_{s} \int d \boldsymbol{k}_{i} F\left(\boldsymbol{k}_{s}, \boldsymbol{k}_{i}\right) \hat{a}^{\dagger}\left(\boldsymbol{k}_{s}\right) \hat{a}^{\dagger}\left(\boldsymbol{k}_{i}\right)|0\rangle,
$$

where $\boldsymbol{k}$ indicates the wave vector and $\hat{a}^{\dagger}(\boldsymbol{k})$ is the creation operator for the mode with $\boldsymbol{k}$ at the crystal. The subscripts $s$, $i$, and $p$ denote the signal, idler, and pump modes. The magnitudes of the wave vectors $\left|\boldsymbol{k}_{j}\right|(j=s, i)$ are given by $\omega_{j} n_{j} / c$, where $n_{j}$ is the refractive index of the crystal, $\omega_{j}$ is the angular frequency, and $c$ is the speed of light. For the type-II SPDC, $n_{i}$ and $n_{s}$ are the refractive indices for the extraordinary-ray and ordinary-ray $\left[n_{i}=n_{i}\left(\boldsymbol{k}_{i}\right)\right.$ and $n_{s}=n_{s}\left(\omega_{s}\right)$, respectively. $F\left(\boldsymbol{k}_{s}, \boldsymbol{k}_{i}\right)$ is the phase-matching function, and $\left|F\left(\boldsymbol{k}_{s}, \boldsymbol{k}_{i}\right)\right|^{2}$ indicates the creation probability of the generated photon pairs with $\boldsymbol{k}_{s}$ and $\boldsymbol{k}_{i} \cdot\left|F\left(\boldsymbol{k}_{s}, \boldsymbol{k}_{i}\right)\right|^{2}$ is given by $[13,14,17]$

$$
\left|F\left(\boldsymbol{k}_{s}, \boldsymbol{k}_{i}\right)\right|^{2}=\delta\left(\omega_{s}+\omega_{i}-\omega_{p}\right) h_{t r}\left(\Delta k_{x}, \Delta k_{y}\right) h\left(\Delta k_{z}\right),
$$

where

$$
\begin{aligned}
& h_{t r}\left(\Delta k_{x}, \Delta k_{y}\right)=\exp \left(-\frac{1}{2} w^{2}\left(\Delta k_{x}^{2}+\Delta k_{y}^{2}\right)\right), \\
& h\left(L \Delta k_{z}\right)=\left[\sin \left(\frac{1}{2} L \Delta k_{z}\right) / \frac{1}{2} L \Delta k_{z}\right]^{2} .
\end{aligned}
$$

$\Delta k_{x}, \Delta k_{y}$, and $\Delta k_{z}$ are the $x, y$, and $z$ components of $\Delta \boldsymbol{k}$ $\left(=\boldsymbol{k}_{s}+\boldsymbol{k}_{i}-\boldsymbol{k}_{p}\right)$, respectively. The function $\delta\left(\omega_{s}+\omega_{i}-\omega_{p}\right)$ indicates energy conservation.
Using Eq. (2), we define the event probability of the creation of single photons, $P\left(\boldsymbol{k}_{s}\right)$, emitted in the direction of $\boldsymbol{k}_{s}$ with frequency $\omega_{s}$. The biphoton probability amplitude of photons with $\left(\boldsymbol{k}_{s}, \omega_{s}\right)$ and $\left(\boldsymbol{k}_{i}, \omega_{i}\right)$ can be written as

$$
\left\langle 0\left|\hat{a}\left(\boldsymbol{k}_{i}\right) \hat{a}\left(\boldsymbol{k}_{s}\right)\right| \psi\right\rangle=F\left(\boldsymbol{k}_{s}, \boldsymbol{k}_{i}\right) .
$$

The event probability of the creation of signal photons can be defined by integrating over all combinations of pair photons with $\boldsymbol{k}_{i}$ in path $i$,

$$
P\left(\boldsymbol{k}_{s}\right)=\int d \boldsymbol{k}_{i}\left|\left\langle 0\left|\hat{a}\left(\boldsymbol{k}_{i}\right) \hat{a}\left(\boldsymbol{k}_{s}\right)\right| \psi\right\rangle\right|^{2}=\int d \boldsymbol{k}_{i}\left|F\left(\boldsymbol{k}_{s}, \boldsymbol{k}_{i}\right)\right|^{2} .
$$

Next, the influences of the optical components set in each optical path should be taken into account. We define an annihilation operator with mode $\boldsymbol{k}_{s}$ at the detector, $\hat{b}\left(\boldsymbol{k}_{s}\right)$. The operator is given by [17]

$$
\hat{b}\left(\boldsymbol{k}_{s}\right)=\sqrt{\eta_{s}} t_{\text {filter }}\left(\omega_{s}\right) t_{\text {iris }}\left(\boldsymbol{k}_{s}\right) \hat{a}\left(\boldsymbol{k}_{s}\right) .
$$

Here, $\eta_{s}$ indicates the quantum efficiency of a photodetector, $t_{\text {filter }}\left(\omega_{s}\right)$ is the amplitude transmittance of the frequency filter, and $t_{\text {iris }}\left(\boldsymbol{k}_{s}\right)$ indicates the amplitude transmittance of the iris, the center of which is set at $\boldsymbol{k}_{\text {iris }}$ with an acceptance angle of $\Delta_{s}$ and a symmetrical profile around the iris center. For simplification, we have assumed that the frequency filter is angle independent, while the iris is frequency independent. In order to evaluate the individual single-photon detection probability $S\left(\boldsymbol{k}_{s}\right)$ after passing through the optical components in path $s$, we replace $\hat{a}\left(\boldsymbol{k}_{s}\right)$ in Eq. (6) with $\hat{b}\left(\boldsymbol{k}_{s}\right)$,

$$
\begin{aligned}
S\left(\boldsymbol{k}_{s}\right) & =\int d \boldsymbol{k}_{i}\left|\left\langle 0\left|\hat{a}\left(\boldsymbol{k}_{i}\right) \hat{b}\left(\boldsymbol{k}_{s}\right)\right| \psi\right\rangle\right|^{2} \\
& =\eta_{s} \xi_{s}\left(\omega_{s}\right) \zeta_{s}\left(\boldsymbol{k}_{s}\right) \int d \boldsymbol{k}_{i}\left|F\left(\boldsymbol{k}_{s}, \boldsymbol{k}_{i}\right)\right|^{2} \\
& =\eta_{s} \xi_{s}\left(\omega_{s}\right) \zeta_{s}\left(\boldsymbol{k}_{s}\right) P\left(\boldsymbol{k}_{s}\right),
\end{aligned}
$$

where the influence of the frequency filter and the iris set in path $s$ can be taken as the intensity transmittance functions $\xi_{s}\left(\omega_{s}\right)=\left|t_{\text {filter }}\left(\omega_{s}\right)\right|^{2}$ and $\zeta_{s}\left(\boldsymbol{k}_{s}\right)=\left|t_{\text {iris }}\left(\boldsymbol{k}_{s}\right)\right|^{2}$, respectively. Thus we find that the detection probability $S\left(\boldsymbol{k}_{s}\right)$ is equivalent to the event probability $P\left(\boldsymbol{k}_{s}\right)$ filtered by the intensity transmittance functions of the optical components in path $s$.

Next, we discuss the conditional single-photon detection probability $C\left(\boldsymbol{k}_{s}\right)$ of a signal photon with $\boldsymbol{k}_{s}$ when the corresponding idler photon is detected by Detector i. $C\left(\boldsymbol{k}_{s}\right)$ can be derived from the replacement of the remaining $\hat{a}\left(\boldsymbol{k}_{i}\right)$ in Eq. (8) with $\hat{b}\left(\boldsymbol{k}_{i}\right)$,

$$
\begin{aligned}
C\left(\boldsymbol{k}_{s}\right) & =\int d \boldsymbol{k}_{i}\left|\left\langle 0\left|\hat{b}\left(\boldsymbol{k}_{i}\right) \hat{b}\left(\boldsymbol{k}_{s}\right)\right| \psi\right\rangle\right|^{2} \\
& =\eta_{s} \xi_{s}\left(\omega_{s}\right) \zeta_{s}\left(\boldsymbol{k}_{s}\right) \int d \boldsymbol{k}_{i} \eta_{i} \xi_{i}\left(\omega_{i}\right) \zeta_{i}\left(\boldsymbol{k}_{i}\right)\left|F\left(\boldsymbol{k}_{s}, \boldsymbol{k}_{i}\right)\right|^{2} .
\end{aligned}
$$

This probability reads the creation probability of the pair photons $\left|F\left(\boldsymbol{k}_{s}, \boldsymbol{k}_{i}\right)\right|^{2}$ filtered by the transmittance functions of the optical components in both paths $s$ and $i$. 
Note that $S\left(\boldsymbol{k}_{s}\right)$ and $C\left(\boldsymbol{k}_{s}\right)$ can be easily obtained by applying the transmittance functions (filters) to $\left|F\left(\boldsymbol{k}_{s}, \boldsymbol{k}_{i}\right)\right|^{2}$ (tuning curve). Similarly, the probabilities $P\left(\boldsymbol{K}_{i}\right), S\left(\boldsymbol{k}_{i}\right)$, and $C\left(\boldsymbol{k}_{i}\right)$ can be found by exchanging subscripts $s$ and $i$.

In order to test the proposed method, we performed experiments using a setup that is similar to the model in Fig. 1. We measured the acceptance-angle-dependent single and coincidence counting rates, $R_{1}\left(\Delta_{s}\right)$ and $R_{c}\left(\Delta_{s}, \Delta_{i}\right)$, while varying the iris size set in each path. $R_{1}\left(\Delta_{s}\right)$ is derived by integrating over $\boldsymbol{k}_{s}$ in Eq. (8),

$$
R_{1}\left(\Delta_{s}\right)=\int d \boldsymbol{k}_{s} \int d \boldsymbol{k}_{i}\left|\left\langle 0\left|\hat{a}\left(\boldsymbol{k}_{i}\right) \hat{b}_{s}\left(\boldsymbol{k}_{s}\right)\right| \psi\right\rangle\right|^{2}=\int d \boldsymbol{k}_{s} S\left(\boldsymbol{k}_{s}\right) .
$$

We can also derive $R_{1}\left(\Delta_{i}\right)$ by exchanging the subscripts $s$ and $i$.

Similarly, $R_{c}\left(\Delta_{s}, \Delta_{i}\right)$ is derived by integrating over $\boldsymbol{k}_{s}$ in Eq. (9),

$$
R_{c}\left(\Delta_{s}, \Delta_{i}\right)=\int d \boldsymbol{k}_{s} \int d \boldsymbol{k}_{i}\left|\left\langle 0\left|\hat{b}\left(\boldsymbol{k}_{i}\right) \hat{b}\left(\boldsymbol{k}_{s}\right)\right| \psi\right\rangle\right|^{2}=\int d \boldsymbol{k}_{s} C\left(\boldsymbol{k}_{s}\right),
$$

which is the integration of all creation probabilities $\left|F\left(\boldsymbol{k}_{s}, \boldsymbol{k}_{i}\right)\right|^{2}$ of photon pairs with $\left(\boldsymbol{k}_{s}, \omega_{s}\right)$ and $\left(\boldsymbol{k}_{i}, \omega_{i}\right)$ filtered by the transmittance functions in both paths. Note that, once $\left|F\left(\boldsymbol{k}_{s}, \boldsymbol{k}_{i}\right)\right|^{2}$ is obtained, all detection probabilities $[P(\boldsymbol{k}), S(\boldsymbol{k})$, and $C(\boldsymbol{k})]$ and acceptance-angle-dependent counting probabilities $\left[R_{1}\left(\Delta_{s}\right), R_{1}\left(\Delta_{i}\right), R_{c}\left(\Delta_{s}, \Delta_{i}\right)\right]$ can be calculated.

\section{B. Calculation of the creation probability using spherical coordinates}

In this section, based partly on Refs. $[13,14,17]$, we consider $\left|F\left(\boldsymbol{k}_{s}, \boldsymbol{k}_{i}\right)\right|^{2}$ with an arbitrary wave vector $\boldsymbol{k}_{i}$ and a fixed wave vector $\boldsymbol{k}_{s}\left(=\boldsymbol{k}_{s}^{0}\right)$ using spherical coordinates. It is convenient to choose the central idler wave vector $\boldsymbol{k}_{\dot{0}}^{0}$, which satisfies the perfect phase-matching condition $\left(\boldsymbol{k}_{s}^{0}+\boldsymbol{k}_{i}^{0}-\boldsymbol{k}_{p}\right.$ $=0)$ and energy conservation $\left(\omega_{s}+\omega_{i}=\omega_{p}\right)$ (Fig. 2). In spherical coordinates, wave vectors can be defined by the polar angle $\theta$ measured from the $z$ axis, the azimuthal angle $\phi$ from the $x$ axis in the $x-y$ plane, and the frequency $\omega$. The wave vectors $\boldsymbol{k}_{s}^{0}$ and $\boldsymbol{k}_{i}^{0}$ are determined by $\left(\omega_{s}, \theta_{s}^{0}, \phi_{s}^{0}\right)$ and $\left(\omega_{i}, \theta_{i}^{0}, \phi_{i}^{0}=\phi_{s}^{0}+\pi\right)$, where $\theta_{s}^{0}, \phi_{s}^{0}, \theta_{i}^{0}$, and $\phi_{i}^{0}$ are the spherical coordinates shown in Fig. 2. $\theta_{i}$ and $\phi_{i}$ are the spherical coordinates for the direction $\boldsymbol{k}_{i}$. For the given $\boldsymbol{k}_{s}^{0}$, the components $\Delta k_{x}, \Delta k_{y}$, and $\Delta k_{z}$ of the phase mismatch $\Delta \boldsymbol{k}$ inside the crystal are given by

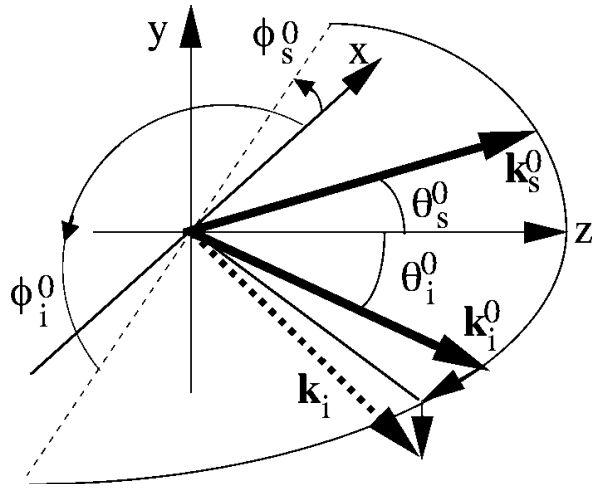

FIG. 2. Coordinates used in the calculation of SPDC.

$$
\begin{gathered}
\Delta k_{x}=\left|\boldsymbol{k}_{i}\right| \sin \theta_{i} \cos \phi_{i}+\left|\boldsymbol{k}_{s}\right| \sin \theta_{s}^{0} \cos \phi_{s}^{0}, \\
\Delta k_{y}=\left|\boldsymbol{k}_{i}\right| \sin \theta_{i} \sin \phi_{i}+\left|\boldsymbol{k}_{s}\right| \sin \theta_{s}^{0} \sin \phi_{s}^{0}, \\
\Delta k_{z}=\left|\boldsymbol{k}_{i}\right| \cos \theta_{i}+\left|\boldsymbol{k}_{s}\right| \cos \theta_{s}^{0}-k_{p} .
\end{gathered}
$$

After a simple calculation, $\Delta k_{\perp}^{2}=\Delta k_{x}^{2}+\Delta k_{y}^{2}$ in Eq. (3) is written as

$$
\begin{aligned}
\Delta k_{\perp}^{2}= & \left|\boldsymbol{k}_{i}\right|^{2} \sin ^{2} \theta_{i}+\left|\boldsymbol{k}_{s}\right|^{2} \sin ^{2} \theta_{s}^{0} \\
& -2\left|\boldsymbol{k}_{s}\right|\left|\boldsymbol{k}_{i}\right| \sin \theta_{i} \sin \theta_{s}^{0} \cos \left(\phi_{i}-\phi_{s}^{0}-\pi\right) .
\end{aligned}
$$

In order to calculate the refractive index $n_{i}$ for the extraordinary ray in the direction of $\boldsymbol{k}_{i}$, we must solve the optical indicatrix equation [14]. Since the optical axis of the crystal is considered to lie in the $x-z$ plane, $n_{i}$ is given by

$$
n_{i}=\sqrt{1 /\left[S_{z}^{2} / n_{o}^{2}+\left(1-S_{z}^{2}\right) / n_{e}^{2}\right]},
$$

where

$$
\begin{aligned}
S_{z}^{2}= & \sin ^{2} \theta_{i} \cos ^{2} \phi_{i} \sin ^{2} \theta_{p}+\cos ^{2} \theta_{i} \cos ^{2} \theta_{p} \\
& -2 \sin \theta_{i} \cos \theta_{i} \cos \phi_{i} \sin \theta_{p} \cos \theta_{p} .
\end{aligned}
$$

$\theta_{p}$ indicates the direction of the optical axis of the crystal. $n_{o}=n_{o}(\omega)$ and $n_{e}=n_{e}(\omega)$ indicate the principal refractive indices of the crystal at a given wavelength and can be calculated from the Sellmeier equations [14]. Note that $n_{s}$ and $\left|\boldsymbol{k}_{s}\right|$ are functions of $\left(\omega_{s}, \theta_{p}\right)$, and $n_{i}$ and $\left|\boldsymbol{k}_{i}\right|$ are functions of $\left(\omega_{i}, \theta_{i}, \phi_{i}, \theta_{p}\right)$.

Substituting $\Delta k_{\perp}$ and $\Delta k_{z}$ into Eqs. (2)-(4), we find that $\left|F\left(\boldsymbol{k}_{s}, \boldsymbol{k}_{i}\right)\right|^{2}$ can be rewritten as a function of $\left(\omega_{i}, \theta_{i}, \phi_{i}\right)$ for the given $\left(\omega_{s}, \theta_{s}^{0}, \phi_{s}^{0}\right)$ as follows:

$$
\begin{aligned}
\left|F\left(\omega_{s}, \theta_{s}^{0}, \phi_{s}^{0}, \omega_{i}, \theta_{i}, \phi_{i}\right)\right|^{2}= & \delta\left(\omega_{p}-\omega_{i}-\omega_{s}\right) \exp \left(-\frac{1}{2} w^{2}\left[\left(\omega_{i} n_{i} / c\right)^{2} \sin ^{2} \theta_{i}+\left(\omega_{s} n_{s} / c\right)^{2} \sin ^{2} \theta_{s}^{0}\right]\right) \exp \left[w^{2}\left(\omega_{s} n_{s} / c\right)\right. \\
& \left.\times\left(\omega_{i} n_{i} / c\right) \sin \theta_{i} \sin \theta_{s}^{0} \cos \left(\phi_{i}-\phi_{s}^{0}-\pi\right)\right]\left[\operatorname{sinc}\left(\frac{1}{2 \pi} L\left[\left(\omega_{i} n_{i} / c\right) \cos \theta_{i}+\left(\omega_{s} n_{s} / c\right) \cos \theta_{s}^{0}-k_{p}\right]\right)\right]^{2}
\end{aligned}
$$


TABLE I. Experimental parameters.

\begin{tabular}{lccccc}
\hline \hline \multicolumn{2}{c}{ Pump beam } & \multicolumn{2}{c}{ Crystal } & \multicolumn{2}{c}{ Frequency filter } \\
diameter $(w)$ & wavelength & thickness $(L)$ & crystal angle $\left(\theta_{p}\right)$ & center wavelength & bandwidth \\
\hline $500 \mu \mathrm{m}$ & $399.6 \mathrm{~nm}$ & $5.0 \mathrm{~mm}$ & $41.44^{\circ}$ & $799.3 \mathrm{~nm}$ & $2.9 \mathrm{~nm}$ \\
\hline \hline
\end{tabular}

Similarly, $P\left(\boldsymbol{k}_{s}\right)$ in Eq. (6) is rewritten as follows:

$$
\begin{aligned}
& P\left(\omega_{s}, \theta_{s}^{0}, \phi_{s}^{0}\right) \\
& \quad=\int_{0}^{\infty} d \omega_{i} \int_{0}^{\pi} d \theta_{i} \int_{0}^{2 \pi} d \phi_{i} J\left|F\left(\omega_{s}, \theta_{s}^{0}, \phi_{s}^{0}, \omega_{i}, \theta_{i}, \phi_{i}\right)\right|^{2} \\
& \quad=\int_{0}^{\pi} d \theta_{i} \int_{0}^{2 \pi} d \phi_{i} J\left|F\left(\omega_{s}, \theta_{s}^{0}, \phi_{s}^{0}, \omega_{p}-\omega_{s}, \theta_{i}, \phi_{i}\right)\right|^{2},
\end{aligned}
$$

where $J$ is the Jacobian for the transformation from $d \boldsymbol{k}_{i}$ to $d \omega_{i} d \theta_{i} d \phi_{i}$. Note that, as a result of energy conservation, the three-dimensional integration in Eq. (6) has been reduced to two-dimensional integration. $S\left(\boldsymbol{k}_{s}\right)$ in Eq. (8) and $C\left(\boldsymbol{k}_{s}\right)$ in Eq. (9) also become as follows:

$$
\begin{aligned}
S\left(\omega_{s}, \theta_{s}^{0}, \phi_{s}^{0}\right)= & \eta_{s} \xi_{s}\left(\omega_{s}\right) \zeta_{s}\left(\theta_{s}^{0}, \phi_{s}^{0}\right) \int_{0}^{\pi} d \theta_{i} \\
& \times \int_{0}^{2 \pi} d \phi_{i} J\left|F\left(\omega_{s}, \theta_{s}^{0}, \phi_{s}^{0}, \omega_{p}-\omega_{s}, \theta_{i}, \phi_{i}\right)\right|^{2} \\
= & \eta_{s} \xi_{s}\left(\omega_{s}\right) \zeta_{s}\left(\theta_{s}^{0}, \phi_{s}^{0}\right) P\left(\omega_{s}, \theta_{s}^{0}, \phi_{s}^{0}\right) \\
C\left(\omega_{s}, \theta_{s}^{0}, \phi_{s}^{0}\right)= & \eta_{s} \xi_{s}\left(\omega_{s}\right) \zeta_{s}\left(\theta_{s}^{0}, \phi_{s}^{0}\right) \int_{0}^{\pi} d \theta_{i} \int_{0}^{2 \pi} d \phi_{i} J \eta_{i} \xi_{i} \\
& \times\left(\omega_{p}-\omega_{s}\right) \zeta_{i}\left(\theta_{i}, \phi_{i}\right) \mid F\left(\omega_{s}, \theta_{s}^{0}, \phi_{s}^{0}, \omega_{p}\right. \\
& \left.-\omega_{s}, \theta_{i}, \phi_{i}\right)\left.\right|^{2},
\end{aligned}
$$

where $\zeta(\boldsymbol{k})$ is denoted as $\zeta(\theta, \phi)$, because $\zeta(\boldsymbol{k})$ is independent of $\omega$.

$R_{1}\left(\Delta_{s}\right)$ in Eq. (10) and $R_{c}\left(\Delta_{s}, \Delta_{i}\right)$ in Eq. (11) can also be rewritten as

$$
\begin{gathered}
R_{1}\left(\Delta_{s}\right)=\int_{0}^{\infty} d \omega_{s} \int_{0}^{\pi} d \theta_{s}^{0} \int_{0}^{2 \pi} d \phi_{s}^{0} J^{\prime} S\left(\omega_{s}, \theta_{s}^{0}, \phi_{s}^{0}\right), \\
R_{c}\left(\Delta_{s}, \Delta_{i}\right)=\int_{0}^{\infty} d \omega_{s} \int_{0}^{\pi} d \theta_{s}^{0} \int_{0}^{2 \pi} d \phi_{s}^{0} J^{\prime} C\left(\omega_{s}, \theta_{s}^{0}, \phi_{s}^{0}\right),
\end{gathered}
$$

where $J^{\prime}$ is also the Jacobian for the transformation from $d \boldsymbol{k}_{s}$ to $d \omega_{s} d \theta_{s}^{0} d \phi_{s}^{0}$. These probabilities for idler photons can be similarly defined by exchanging subscripts $s$ and $i$.

\section{Calculation for the beam-like twin-photons using type-II SPDC [7]}

In order to test the proposed method, we compare the calculated probabilities with the experimental results using a setup similar to the model (Fig. 1). In this section, we explain how to calculate the probabilities using Eqs. (16)-(21) under specific experimental conditions, namely the phasematching condition for the beamlike twin-photon generation [7], which was used in the experiments. The parameters used in the experiments were listed in Table I. Under this condition, the distribution of the emitted photons in each path is estimated to be below $3^{\circ}$ around each central propagation axis of the twin photons, the directions of which are determined by $\left(\theta_{s}^{c} \simeq 2.1^{\circ}, \phi_{s}^{c}=0\right)$ and $\left(\theta_{i}^{c} \simeq 2.1^{\circ}, \phi_{i}^{c}=\pi\right)$ in the crystal (see Fig. 3). The generated photons are distributed symmetrically around each central propagation axis, which is suggested in Ref. [17] and can also be confirmed experimentally [7]. Here, we assume the rotational symmetry for $P\left(\omega_{s}, \theta_{s}^{0}, \phi_{s}^{0}\right), S\left(\omega_{s}, \theta_{s}^{0}, \phi_{s}^{0}\right)$, and $C\left(\omega_{s}, \theta_{s}^{0}, \phi_{s}^{0}\right)$ around each central propagation axis. Thus we first calculate $P\left(\omega_{s}, \theta_{s}^{0}, \phi_{s}^{0}\right), S\left(\omega_{s}, \theta_{s}^{0}, \phi_{s}^{0}\right)$, and $C\left(\omega_{s}, \theta_{s}^{0}, \phi_{s}^{0}\right)$ in the $x$-z plane $\left(\phi_{s}^{0}=0\right)$. From these distributions, $R_{1}\left(\Delta_{s}\right)$ and $R_{c}\left(\Delta_{s}, \Delta_{i}\right)$ are calculated taking the rotational symmetry into account.

Let us consider the integration over $\phi_{i}$ in this situation. In Eq. (16), $n_{i}$ and the third term, including $\cos \left(\phi_{i}-\phi_{s}^{0}-\pi\right)$, depend on the parameter $\phi_{i}$. With the experimental parameters, the change in $n_{i}$ along the azimuthal direction $\phi_{i}$ is estimated to be less than $3 \%$. Therefore we neglect the $\phi_{i}$ dependence of $n_{i}$ in order to simplify the calculation. For the third term, the result of the integration over $\phi_{i}$ is as follows:

$$
\int_{0}^{2 \pi} d \phi_{i} \exp \left[a\left(\theta_{i}\right) \cos \left(\phi_{i}-\pi\right)\right]=2 \pi I_{0}\left(a\left(\theta_{i}\right)\right)
$$

where

$$
a\left(\theta_{i}\right)=w^{2}\left(\omega_{s} n_{s} / c\right)\left(\omega_{i} n_{i} / c\right) \sin \theta_{i} \sin \theta_{s}^{0}
$$

and the function $I_{0}$ indicates the modified Bessel function of the first kind of order 0 . If $a\left(\theta_{i}\right)$ is not close to 0 , which means that $\theta_{i}$ and $\theta_{s}^{0}$ are not close to $0^{\circ}, I_{0}\left(a\left(\theta_{i}\right)\right)$ can be simplified. From Ref. [18], when $a\left(\theta_{i}\right)$ is larger than 3.75, $I_{0}\left(a\left(\theta_{i}\right)\right)$ can be well approximated as

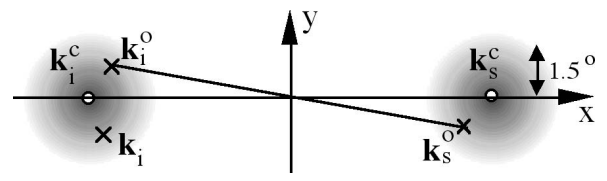

FIG. 3. Typical distribution of type-II beamlike photon-pair generation with a narrow-band filter. 


$$
\begin{aligned}
I_{0}\left(a\left(\theta_{i}\right)\right) \simeq & \frac{1}{\sqrt{2 \pi a\left(\theta_{i}\right)}} \exp \left[a\left(\theta_{i}\right)\right]\left[1+0.03 / a\left(\theta_{i}\right)\right. \\
& \left.+O\left(1 / a\left(\theta_{i}\right)^{2}\right)\right] .
\end{aligned}
$$

This equation can be further simplified for a larger value of $a\left(\theta_{i}\right)$. When $\theta_{i}^{0}$ is sufficiently far from $0^{\circ}\left(\theta_{i}^{0} \geqslant 0.03^{\circ}\right)$, the components with $\theta_{i}$ that consistently satisfies the condition of $a\left(\theta_{i}\right) \geqslant 100$ only contribute to the numerical results [19]. Therefore when $\theta_{i}^{0} \geqslant 0.03^{\circ},\left|F\left(\omega_{s}, \theta_{s}^{0}, \phi_{s}^{0}=0, \omega_{i}, \theta_{i}, \phi_{i}\right)\right|^{2}$ after the integration over $\phi_{i}$ is well approximated by

$$
\begin{aligned}
\int_{0}^{2 \pi} d & \phi_{i} \mid F\left(\omega_{s}, \theta_{s}^{0}, \phi_{s}^{0}\right. \\
= & \left.0, \omega_{i}, \theta_{i}, \phi_{i}\right)\left.\right|^{2} \simeq \frac{\delta\left(\omega_{p}-\omega_{i}-\omega_{s}\right)}{\sqrt{2 \pi a\left(\theta_{i}\right)}} \\
& \times \exp \left(-\frac{1}{2} w^{2}\left[\left(\omega_{i} n_{i} / c\right) \sin \theta_{i}-\left(\omega_{s} n_{s} / c\right) \sin \theta_{s}^{0}\right]^{2}\right) \\
& \times\left[\operatorname{sinc}\left(\frac{1}{2 \pi} L\left[\left(\omega_{i} n_{i} / c\right) \cos \theta_{i}+\left(\omega_{s} n_{s} / c\right) \cos \theta_{s}^{0}-k_{p}\right]\right)\right]^{2} \\
\operatorname{def} & \left|\widetilde{F}\left(\omega_{s}, \theta_{s}^{0}, \omega_{i}, \theta_{i}\right)\right|^{2} .
\end{aligned}
$$

According to these results, $P\left(\omega_{s}, \theta_{s}^{0}, \phi_{s}^{0}\right)$ in the $x$-z plane [Eq. (17)] is simply given by

$$
\begin{aligned}
P\left(\omega_{s}, \theta_{s}^{0}, \phi_{s}^{0}=0\right) & =N \int_{0}^{\pi} d \theta_{i} J\left|\tilde{F}\left(\omega_{s}, \theta_{s}^{0}, \omega_{p}-\omega_{s}, \theta_{i}\right)\right|^{2} \\
& \operatorname{def} \\
& =P\left(\omega_{s}, \theta_{s}^{0}\right),
\end{aligned}
$$

where $N$ is the normalization constant. Using Eqs. (25) and (26), $S\left(\omega_{s}, \theta_{s}^{0}, \phi_{s}^{0}\right)$ and $C\left(\omega_{s}, \theta_{s}^{0}, \phi_{s}^{0}\right)$ in the $x$ - $z$ plane [Eqs. (18) and (19)] are also written as

$$
S\left(\omega_{s}, \theta_{s}^{0}, \phi_{s}^{0}=0\right)=\eta_{s} \xi_{s}\left(\omega_{s}\right) \zeta_{s}\left(\theta_{s}^{0}\right) P\left(\omega_{s}, \theta_{s}^{0}\right) \stackrel{\text { def }}{=} S\left(\omega_{s}, \theta_{s}^{0}\right),
$$

$$
\begin{aligned}
C\left(\omega_{s}, \theta_{s}^{0}, \phi_{s}^{0}=0\right)= & N \eta_{s} \xi_{s}\left(\omega_{s}\right) \zeta_{s}\left(\theta_{s}^{0}\right) \int_{0}^{\pi} d \theta_{i} J \eta_{i} \xi_{i}\left(\omega_{p}\right. \\
& \left.\quad-\omega_{s}\right) \zeta_{i}\left(\theta_{i}\right) \times\left|\tilde{F}\left(\omega_{s}, \theta_{s}^{0}, \omega_{p}-\omega_{s}, \theta_{i}\right)\right|^{2} \\
& \operatorname{def} \\
= & C\left(\omega_{s}, \theta_{s}^{0}\right),
\end{aligned}
$$

where $\zeta_{s}\left(\theta_{s}\right)$ and $\zeta_{i}\left(\theta_{i}\right)$ were assumed to be rectangular step functions ( 1 for inside the iris and 0 for outside the iris) with the point of symmetry.

Under the assumption that the generated photons are distributed symmetrically around each central propagation axis, $R_{1}\left(\Delta_{s}\right)$ in Eq. (20) and $R_{c}\left(\Delta_{s}, \Delta_{i}\right)$ in Eq. (21) can be calculated using $S\left(\omega_{s}, \theta_{s}^{0}\right)$ and $C\left(\omega_{s}, \theta_{s}^{0}\right)$, as follows:

$$
R_{1}\left(\Delta_{s}\right)=N \int_{0}^{\pi} d \theta_{s}^{0} 2 \pi\left|\theta_{s}^{0}-\theta_{s}^{c}\right|\left(\int_{0}^{\infty} d \omega_{s} S\left(\omega_{s}, \theta_{s}^{0}\right)\right),
$$

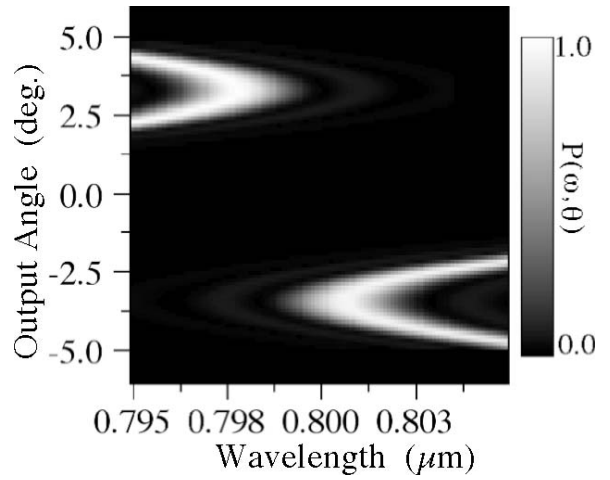

FIG. 4. Event probabilities $P\left(\theta_{s}, \omega_{s}\right)$ (upper curve) and $P\left(\theta_{i}, \omega_{i}\right)$ (lower curve) without the presence of filters or irises calculated from Eq. (26).

$$
R_{c}\left(\Delta_{s}, \Delta_{i}\right)=N \int_{0}^{\pi} d \theta_{s}^{0} 2 \pi\left|\theta_{s}^{0}-\theta_{s}^{c}\right|\left(\int_{0}^{\infty} d \omega_{s} C\left(\omega_{s}, \theta_{s}^{0}\right)\right),
$$

where $\theta_{s}^{c}$ indicates the emission angle of the type-II beamlike twin-photon generation. Those probabilities for idler photons can be similarly defined by exchanging subscripts $s$ and $i$.

\section{Numerical calculation}

In order to perform the numerical calculation, we used the discrete approximation in the $\omega$ and $\theta$ regions, that is, the integration $\int d \omega \int d \theta$ was replaced by $\Sigma_{\omega} \Sigma_{\theta} \delta \omega \delta \theta$ with the intervals of $\delta \omega$ and $\delta \theta$. These intervals were set to be sufficiently smaller than the phase mismatch as determined by the transverse profile of the excitation beam and the crystal thickness. The parameters were set according to the experimental conditions (Table I). $\theta_{s}$ and $\theta_{i}$ were taken from $-3^{\circ}$ to $3^{\circ}$ in the crystal (from $-5.5^{\circ}$ to $5.5^{\circ}$ outside the crystal). $\omega_{s}$ and $\omega_{i}$ were taken from 795 to $805 \mathrm{~nm}$. These wavelengths and angles were divided by 2000 points, which means that $\delta \omega_{s}=\delta \omega_{i}=0.005 \mathrm{~nm}$ and $\delta \theta_{s}=\delta \theta_{i}=0.003^{\circ}$, respectively.

According to Eq. (26), $P\left(\omega_{s}, \theta_{s}\right)$ and $P\left(\omega_{i}, \theta_{i}\right)$ were separately calculated. When $P\left(\omega_{s}, \theta_{s}\right)$ for given $\omega_{s}$ and $\theta_{s}$ was calculated, the value of $J\left|\tilde{F}\left(\omega_{s}, \theta_{s}^{0}, \omega_{p}-\omega_{s}, \theta_{i}\right)\right|^{2}$ in the integration of Eq. (26) was calculated for each $\theta_{i}$. By taking the summation of these calculated values over $\theta_{i}, P\left(\omega_{s}, \theta_{s}\right)$ for given $\omega_{s}$ and $\theta_{s}$ was obtained. The calculation was repeatedly performed for different $\omega_{s}$ and $\theta_{s}$. Similarly, $P\left(\omega_{i}, \theta_{i}\right)$ was calculated.

Figure 4 shows a two-dimensional plot of the event probabilities of the creation of single photons $P\left(\omega_{s}, \theta_{s}\right)$ and $P\left(\omega_{i}, \theta_{i}\right)$ as functions of $\omega$ and $\theta$ for the individual paths, in which $P\left(\omega_{s}, \theta_{s}\right)$ and $P\left(\omega_{i}, \theta_{i}\right)$ are superimposed on the figure. In the figure, $\theta_{i}$ is represented by negative values in order to express the direction of idler photons $\left(\phi_{i}^{0}=\pi\right)$, which is opposite to the direction of signal photons $\left(\phi_{s}^{0}=0^{\circ}\right)$. In addition, $\theta_{s}$ and $\theta_{i}$ are converted into the angles outside the crystal. The profile in Fig. 4 is approximately the same as the calculated results reported in [14]. Note that the calculation is only valid when $|\theta| \geqslant 0.03^{\circ}$, which corresponds to output 


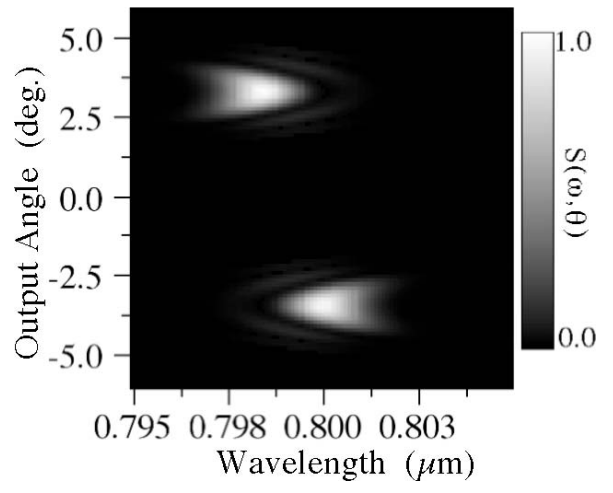

FIG. 5. Detection probabilities $S\left(\omega_{s}, \theta_{s}\right)$ (upper curve) and $S\left(\omega_{i}, \theta_{i}\right)$ (lower curve) after the interference filter in Fig. 6 calculated from Eq. (27).

angles larger than $0.05^{\circ}$. However, with the parameters under consideration, photons are emitted in the valid region $\left(\left|\theta^{\prime}\right|\right.$ $\geqslant 0.05^{\circ}$ ), as shown in Fig. 4.

Figure 5 shows a two-dimensional plot of the individual single-photon detection probabilities $S\left(\omega_{s}, \theta_{s}\right)$ and $S\left(\omega_{i}, \theta_{i}\right)$ after passing through the frequency filter without the irises $\left[\zeta_{s}\left(\theta_{s}\right)=\zeta_{i}\left(\theta_{i}\right)=1\right]$. From Eq. (27), Fig. 5 is considered to be the product of the values in Fig. 4 and the intensity transmittance functions of the frequency filter, $\xi_{s}\left(\omega_{s}\right)$ and $\xi_{i}\left(\omega_{i}\right)$, which were assumed to have the same Gaussian profile, as shown in Fig. 6 and Table I. $\zeta_{s}\left(\theta_{s}\right)$ and $\zeta_{i}\left(\theta_{i}\right)$ were assumed to be set at $\theta_{s}=2.1^{\circ}$ and $\theta_{i}=-2.1^{\circ}$ in the crystal $\left(\theta_{s}=3.35^{\circ}\right.$ and $\theta_{i}=-3.38^{\circ}$ outside the crystal), which corresponds to the emission propagation axis for type-II beamlike twin-photon generation. The quantum efficiency of the photodetector was assumed to be $\eta_{s}=\eta_{i}=1$.

Figure 7 shows a two-dimensional plot of the conditional single-photon detection probabilities $C\left(\omega_{s}, \theta_{s}\right)$ and $C\left(\omega_{i}, \theta_{i}\right)$ calculated using Eq. (28). Here, $C\left(\omega_{s}, \theta_{s}\right)$ means the detection probability of signal photons with $\omega_{s}$ and $\theta_{s}$ when the idler photons through the iris in path $i$ are detected. In the calculation, the iris was assumed to be set only in path $i$ centered at $\theta_{i}=-2.1^{\circ}$ in the crystal $\left(\theta_{i}=-3.38^{\circ}\right.$ outside the crystal) with the acceptance angle $\Delta_{i}=0.43^{\circ}$. The position of the iris in path $i$ almost corresponded to the emission propagation axis for type-II beamlike twin-photon generation. The frequency filters were set in each path. Compared with Fig. 5 , it is clearly seen that the profile of $C\left(\omega_{s}, \theta_{s}\right)$ in Fig. 7 is affected by the iris in path $i$. In other words, the influence of

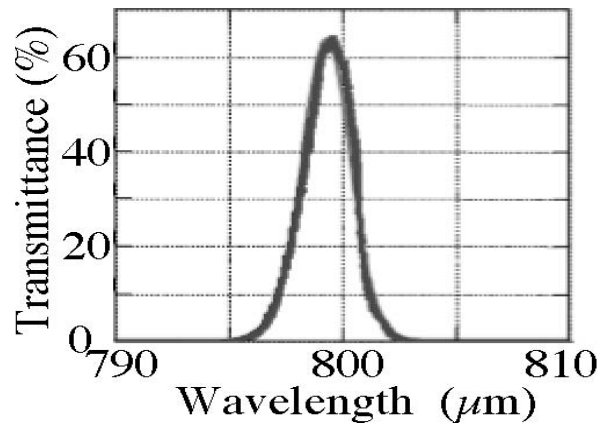

FIG. 6. Transmittance of the interference filter.

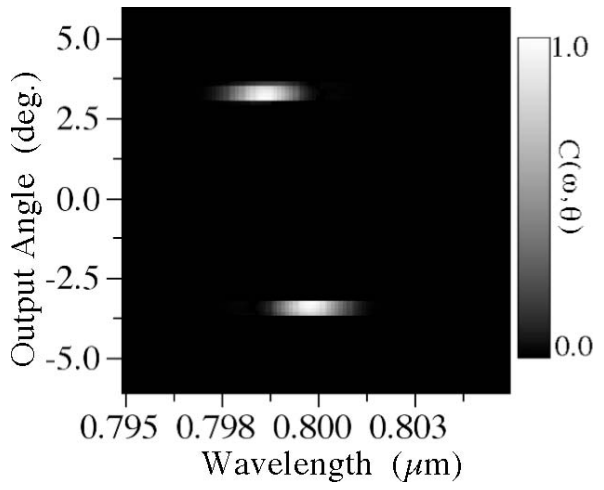

FIG. 7. Conditional single-photon detection probabilities $C\left(\omega_{s}, \theta_{s}\right)$ (upper curve) and $C\left(\omega_{i}, \theta_{i}\right)$ (lower curve) after passing through the interference filter and the iris calculated from Eq. (28). The iris was set in the idler path centered at $-3.38^{\circ}$ with an acceptance angle of $0.43^{\circ}$ (3 $\mathrm{mm}$ diameter at $40 \mathrm{~cm}$ from the crystal).

the iris in path $i$ appears in the detection probability of signal photons in path $s$, where the iris is not set, through the correlation of the phase-matching function. Note that the distribution of $C\left(\omega_{s}, \theta_{s}\right)$ is slightly broader than that of $C\left(\omega_{i}, \theta_{i}\right)$.

We also calculated $R_{1}\left(\Delta_{s}\right)$ and $R_{c}\left(\Delta_{s}, \Delta_{i}\right)$ from Eqs. (29) and (30) using the discrete approximation and calculated $S(\omega, \theta)$ and $C(\omega, \theta)$. In order to compare the calculated results with the experimental data, the details are described in the following section.

\section{EXPERIMENTAL SETUP}

The experimental setup, which is similar to the model in Fig. 1, is shown in Fig. 8. A horizontally polarized second harmonic beam (wavelength: $399.6 \mathrm{~nm}$; power: $50 \mathrm{~mW}$; Gaussian profile with a width of $500 \mu \mathrm{m}$ ) from a ring-cavity SHG unit (MBD-200, Coherent) excited by a tunable CW Ti:sapphire laser (MBR-110, Coherent; wavelength: $799.3 \mathrm{~nm}$, full width at half maximum: $100 \mathrm{kHz}$ ) was used as the excitation light. The excitation light was incident on a BBO crystal $(5 \times 5 \times 5 \mathrm{~mm})$. The angle between the excitation light and the optical axis of the crystal was set to be $41.4^{\circ}$ for the type-II beamlike twin-photon generation [7,9]. The photon pairs had orthogonal polarizations and passed through an interference filter (IF; center wavelength: $799.3 \mathrm{~nm}$, full width at $1 / e^{2}: 2.9 \mathrm{~nm}$, shown in Fig. 6). The excitation light was then cut by dichroic and color filters (F1 and F2). Irises to select the photon-pairs were set at 40 or

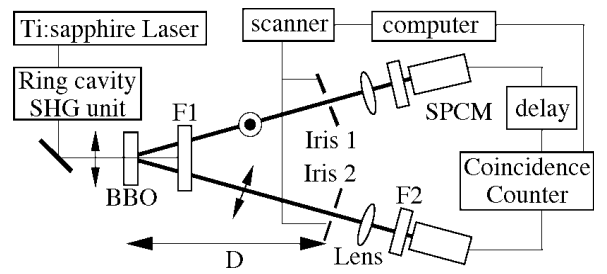

FIG. 8. Experimental setup. F1=interference filter and dichroic mirror, $\mathrm{F} 2=$ sharp cut filters, $\mathrm{D}=$ distance between $\mathrm{BBO}$ and irises, SPCM $=$ single-photon counting module. 

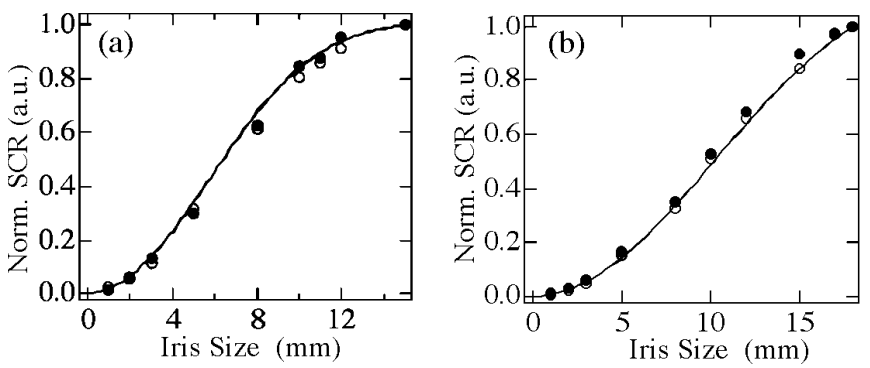

FIG. 9. Iris size dependencies of the single counting rate measured in the signal (circles) and idler (solid circles) paths at distances of (a) 40 and (b) $70 \mathrm{~cm}$, respectively. The photon counting rates were averaged over ten measurements. Statistical errors of the data were less than $3 \%$ of the average value. The solid line indicates the calculated results. These values were normalized by the maximal values with the iris diameters of (a) 15 and (b) $18 \mathrm{~mm}$.

$70 \mathrm{~cm}$ from the crystal in each path, and the center of the irises were adjusted for maximal values of the single and coincidence counting rates. The photon pairs through these optical components were collected and focused onto the active area of the single-photon counting modules (SPCM: SPCM-AQR-14, Perkin-Elmer; quantum efficiency: $\sim 50 \% @ 800 \mathrm{~nm}$ ) by a lens having a focal length of $100 \mathrm{~mm}$. Signals from the SPCMs were sent to a counter (SR400, Stanford Research Systems; gate time: $4 \mathrm{ns),} \mathrm{and}$ the single and coincidence counting rates were measured. The raw counting rates obtained were compensated by the transmittances of the optical components, the background counts, and the quantum efficiencies of the SPCMs. Using a ray tracing method, we confirmed that all of the photons through the optical components were detected by the SPCMs as long as the iris diameter was between 3 and 15 (18) $\mathrm{mm}$ at a distance of $40(70) \mathrm{cm}$ from the crystal.

\section{RESULTS AND DISCUSSION}

Figure 9 shows the single counting rates measured with changing the iris sizes at (a) 40 and (b) $70 \mathrm{~cm}$ from the crystal. Open circles and solid circles indicate the results measured in the signal and idler paths, respectively. First, we used irises with diameters of $3 \mathrm{~mm}$ or less, and adjusted their positions to maximize the single counting rate in each path. We then measured the single counting rates by changing the diameters of individual irises. In Fig. 9(a), the measured single counting rates increase with increasing iris size. When the iris diameter is larger than $12 \mathrm{~mm}$, the single counting rates begin to saturate. In Fig. 9(b), we also find similar behavior, wherein the saturation occurs for iris diameters of over $15 \mathrm{~mm}$.

The solid lines in Fig. 9 indicate the calculated results of $R_{1}\left(\Delta_{s}\right)$ and $R_{1}\left(\Delta_{i}\right)$ using Eq. (29) with the calculated results of $S\left(\omega_{s}, \theta_{s}\right)$ and $S\left(\omega_{i}, \theta_{i}\right)$. After calculating $S\left(\omega_{s}, \theta_{s}\right)$ for given $\Delta_{s}$ in Eq. (27) for each $\omega_{s}$ and $\theta_{s}, R_{1}\left(\Delta_{s}\right)$ was calculated by taking the summation of $S\left(\omega_{s}, \theta_{s}\right)$ over $\theta_{s}$ and $\omega_{s}$ in the range of the integration. Repeatedly calculating $R_{1}\left(\Delta_{s}\right)$ with changing $\Delta_{s}$, the acceptance-angle-dependent single counting probability was calculated. For $R_{1}\left(\Delta_{i}\right)$, a similar calculation

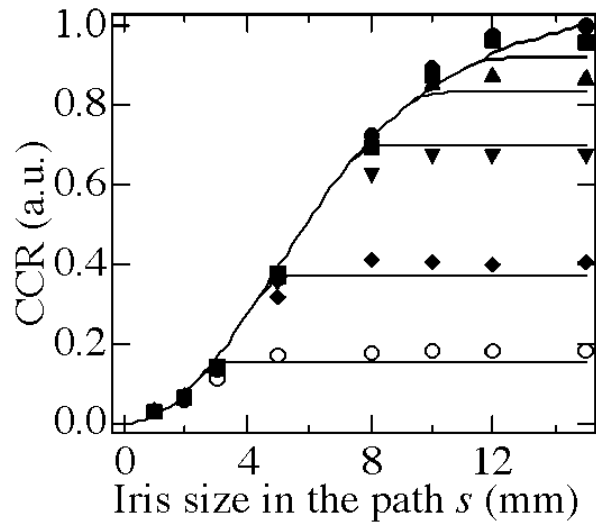

FIG. 10. Iris size dependences of the coincidence counting rate at a distance of $40 \mathrm{~cm}$. The sizes of the iris in the idler path were set to 3 (circles), 5 (solid diamonds), 8 (solid inverted triangles), 10 (solid triangles), 12 (solid squares), and 15 (solid circles) mm, respectively. The lateral axis indicates the iris size in the signal path. The coincidence counting rates were averaged over ten measurements. Statistical errors of the data were less than $4 \%$ of the average value. Solid lines indicate the calculated results obtained by changing the fixed iris size. From bottom to top, the sizes were 3, 5, 8, 10, 12 , and $15 \mathrm{~mm}$. Each of the experimental and numerical data was normalized by the maximal value when both of the iris sizes were set to be $15 \mathrm{~mm}$.

was performed using $S\left(\omega_{i}, \theta_{i}\right)$. In the calculation, $\Delta_{s}$ and $\Delta_{i}$ were taken from 0 to $2.1^{\circ}$ and divided by 100 points. The irises were assumed to be set at $\theta_{i}=-3.38^{\circ}$ and $\theta_{s}=3.35^{\circ}$ outside the crystal, wherein $R_{1}\left(\Delta_{s}\right)$ and $R_{1}\left(\Delta_{i}\right)$ exhibited a maximum in each path. From the calculation of the perfect phase-matching condition, these $\theta_{s}$ and $\theta_{i}$ corresponded approximately to the emission angles for type-II beamlike twin-photon generation. The iris sizes were converted from $\Delta_{j}(j=s, i)$ in the calculation by the simple relation of $\simeq D \tan \left(\Delta_{j}\right)$. Comparing the results at 40 and $70 \mathrm{~cm}$, the calculated results were found to correspond well with the experimental results. The overall behavior, such as the rise at a smaller iris size, the subsequent linear increase, and saturation, can be reproduced without any fitting parameters. It should be noted that the solid line in Fig. 9(b) corresponds to the enlarged view of Fig. 9(a) from 0 to $10 \mathrm{~mm}$. Since the experimental data at 40 and $70 \mathrm{~cm}$ correspond well to the calculated results, we can confirm that the influence of the diffraction effect, which is ignored in the calculation, is sufficiently small.

Next, Fig. 10 shows the iris size dependences of the coincidence counting rates with the iris set at a distance of $40 \mathrm{~cm}$ in each path. First, we used irises with diameters of $3 \mathrm{~mm}$ or less, and adjusted their positions to maximize the coincidence counting rate. We then measured the coincidence counting rate by changing the iris size in the signal path and fixing the iris size in the idler path. For a given fixed size of the iris in the idler path, the coincidence counting rate initially becomes larger as the size of the iris in the signal path is increased. The coincidence counting rates then saturate at a certain iris size. The size at which the saturation begin is approximately the same as the size of the opposite iris. This behavior is observed for all sizes of the iris in the 
idler path. Solid lines in Fig. 10 indicate the calculated results of $R_{c}\left(\Delta_{s}, \Delta_{i}\right)$ in Eq. (30). The procedure was similar to that for $R_{1}\left(\Delta_{s}\right)$. The difference was the use of $C(\omega, \theta)$ instead of $S(\omega, \theta)$. In the calculation, we calculated $R_{c}\left(\Delta_{s}, \Delta_{i}\right)$ for six different sizes of the iris in path $i$ (see Fig. 10). $\Delta_{s}$ was taken from 0 to $2.1^{\circ}$ and divided by 100 points. The irises in each path were assumed to be set at $-3.38^{\circ}$ in path $i$ and $3.35^{\circ}$ in path $s$. For a given fixed size of the iris in path $i, R_{c}\left(\Delta_{s}, \Delta_{i}\right)$ becomes larger with increasing size of the iris in path $s$. The probability then saturates at a certain iris size, which is approximately the same as the size of the iris in path $i$. Similar behaviors are observed for all of the iris sizes in path $i$. These behaviors can also be explained by the two-photon geometric optics proposed by Pittman et al. [10], where they suggested that an out-of-focus image of the fixed iris was projected onto the opposite iris via the correlation of the photon pairs.

From the above results, we can conclude that, using the proposed method, the coincidence counting rates as well as the single counting rates can be reproduced without any fitting parameters, except for the normalization. In addition, the assumption of the symmetrical distribution of the generated photons is valid under the given conditions. The slight disagreements between the numerical results and the experimental data may be attributed primarily to the systematic errors in the experiment, such as the size of the irises, the distances of the irises from the crystal, and the position of the iris center.

\section{CONCLUSIONS}

We proposed the tuning-curve filtering method to evaluate the propagation of photon pairs through the optical components, in which the influence of the optical components set in the propagation axis of photon pairs can be analyzed by applying the transmittance functions of the optical components to the tuning curves. In this method, once the phasematching function $F\left(\boldsymbol{k}_{s}, \boldsymbol{k}_{i}\right)$ is obtained, the propagation of photon pairs through optical components can be calculated. Comparing with the experimental results, we confirmed that the calculated results using this method reproduced the experimental results of the single counting rates and the coincidence counting rates. Since this result indicates that the proposed method is valid, we can analyze the propagation of the photon pairs through arbitrary optical components by using the tuning-curve filtering method. Finally, we briefly note that the proposed method can also be applied to other phasematching conditions, such as sources of Kwiat et al. [6], using Eqs. (17)-(21), although a large amount of computational power may be required.

\section{ACKNOWLEDGMENTS}

The authors would like to thank S. Alexandre and R. Okamoto for their useful discussions.
[1] C. H. Bennett, G. Brassard, C. Crepeau, R. Jozsa, A. Peres, and W. K. Wootters, Phys. Rev. Lett. 70, 1895 (1993).

[2] D. Gottesman and I. L. Chuang, Nature (London) 401, 390 (1999).

[3] S. Takeuchi, Phys. Rev. A 61, 052302 (2000).

[4] C. K. Hong and L. Mandel, Phys. Rev. Lett. 56, 58 (1986).

[5] A. N. Boto, P. Kok, D. S. Abrams, S. L. Braunstein, C. P. Williams, and J. P. Dowling, Phys. Rev. Lett. 85, 2733 (2000).

[6] P. G. Kwiat, K. Mattle, H. Weinfurter, A. Zeilinger, A. V. Sergienko, and Y. Shih, Phys. Rev. Lett. 75, 4337 (1995).

[7] S. Takeuchi, Opt. Lett. 26, 843 (2001).

[8] T. B. Pittman, B. C. Jacobs, and J. D. Franson, Opt. Commun. 246, 545 (2005).

[9] Y.-H. Kim, Phys. Rev. A 68, 013804 (2003).

[10] T. B. Pittman, D. V. Strekalov, D. N. Klyshko, M. H. Rubin, A. V. Sergienko, and Y. H. Shih, Phys. Rev. A 53, 2804 (1996).

[11] T. B. Pittman, Y. H. Shih, D. V. Strekalov, and A. V. Sergienko, Phys. Rev. A 52, R3429 (1995).

[12] A. Joobeur, B. E. A. Saleh, and M. C. Teich, Phys. Rev. A 50, 3349 (1994).

[13] A. Joobeur, B. E. A. Saleh, T. S. Larchuk, and M. C. Teich, Phys. Rev. A 53, 4360 (1996).
[14] N. Boeuf, D. Branning, I. Chaperot, E. Dauler, S. Guérin, G. Jaeger, A. Muller, and A. Migdall, Opt. Eng. (Bellingham) 39, 1016 (2000).

[15] Y. Kawabe, H. Fujiwara, S. Takeuchi, and K. Sasaki, Meeting Abstracts of the Phys. Soc. of Japan 59, 185 (2004).

[16] C. Kurtsiefer, M. Oberparleiter, and H. Weinfurter, J. Mod. Opt. 48, 1997 (2001).

[17] M. H. Rubin, Phys. Rev. A 54, 5349 (1996).

[18] W. H. Press, B. P. Flannery, S. A. Teukolsky, and W. T. Vetterling, Numerical Recipes in FORTRAN (Cambridge University Press, Cambridge, England, 1992).

[19] For $a\left(\theta_{i}\right) \geqslant 100$, the terms $0.03 / a\left(\theta_{i}\right)$ and $O\left[1 / a\left(\theta_{i}\right)^{2}\right]$ become less than $10^{-3}$ and are negligible compared to the principle term 1. The condition $a\left(\theta_{i}\right) \geqslant 100$ is satisfied when $\theta_{i}$ $\geqslant 0.004^{\circ}$. In order to consistently satisfy the condition $\theta_{i}$ $\geqslant 0.004^{\circ}, \quad\left|\theta_{i}^{0}\right|$ has to be larger than $0.03^{\circ}$, because $\left|F\left(\omega_{s}, \theta_{s}^{0}, \phi_{s}^{0}=0, \omega_{i}, \theta_{i}, \phi_{i}\right)\right|^{2}$ for given $\left(\omega_{s}, \theta_{s}\right)$ has considerable value only when $\left|\theta_{i}-\theta_{i}^{0}\right| \leqslant 0.03^{\circ}$ and $\left|\phi_{i}-\phi_{i}^{0}\right| \leqslant 0.6^{\circ}$. Therefore when $\theta_{i}^{0}$ is sufficiently far from $0^{\circ}\left(\theta_{i}^{0} \geqslant 0.03^{\circ}\right)$, there is no contribution to the numerical results from the components with $\theta_{i} \leqslant 0.004^{\circ}$. 